Acta Crystallographica Section D

Biological

Crystallography

ISSN 0907-4449

\section{Commission on Biological Macromoleculest}

+ Correspondence should be addressed to Mitchell Guss, Chairman of the Commission on Biological Macromolecules, Department of Biochemistry, University of Sydney, NSW 2006, Australia, e-mail: m.guss@biochem.usyd.edu.au.

\title{
Guidelines for the deposition and release of macromolecular coordinate and experimental data
}

Recent discussions of the Commission on Biological Macromolecules have confirmed support for a modification of the existing guidelines for the deposition and release of macromolecular coordinate and experimental data.

Consequently, the IUCr has adopted the recommendations of Baker \& Saenger [Baker \& Saenger (1999), Acta Cryst. D55, 2-3], with minor modifications. We recognize that the over-riding motivation is to ensure that the data is deposited and that it will be freely available to the scientific community. We are also aware of the fact that there is a strong feeling that some delay on release of the data after publication may be warranted in exceptional circumstances. These ethical issues are extensively canvassed in the Baker and Saenger article.

The modifications to the published statement concern the following points.

(a) Namely that structure-factor amplitudes be treated in the same way as coordinates. That is, a maximum delay of six months from publication may be requested but that normally they should be available from publication of the relevant article.

(b) That publication of ID codes be modified to indicate relevant PDB or NDB entry codes.

The full text of the modified recommendations is as follows.

\section{Recommendations}

\section{Deposition}

All publications that describe macromolecular three-dimensional structures at the level of individual atomic positions must be accompanied by deposition of both the atomic coordinates and the structure-factor amplitudes in the appropriate database (PDB or NDB). In the case of low-resolu- tion structures for which only a chain trace is reported a set of $\mathrm{C} \alpha$ positions and structurefactor amplitudes may be sufficient.

\section{Publication of ID codes}

An article should not finally be accepted for publication by a journal until the relevant PDB or NDB entry codes have been provided. These entry codes should be quoted in both the text and the abstract (or article header information) so that abstracting services will access them. Journals are also urged to check that the appropriate data have actually been deposited, as part of the process of final acceptance.

\section{Release}

Authors are urged to release the atomic parameters and structure-factor amplitudes immediately after the publication date. This should be the normal practice. They can, however, request a delay of up to six months in the release of the atomic parameter data and the structure-factor amplitudes.

\section{Other aspects}

The IUCr recognizes the principle that the results of publicly funded research should be publicly available. While this is a matter for the funding bodies in different countries, the IUCr encourages crystallographers to put pressure on funding bodies in the public domain to insist on deposition of atomic parameters and structure-factor amplitudes and to require release at time of publication. The IUCr also urges crystallographers to use their influence to ensure that all journals that publish articles on macromolecular three-dimensional structure require the deposition of both atomic parameters and structure-factor amplitudes. 